\title{
LncRNA MM2P-induced, exosome-mediated transfer of Sox9 from monocyte-derived cells modulates primary chondrocytes
}

Jinyu Bai', Yingzi Zhang ${ }^{1}$, Xin Zheng ${ }^{1}$, Man Huang ${ }^{2}$, Weinan Cheng ${ }^{1}$, Huajian Shan', Xiang Gao ', Mingchao Zhang', Lei Sheng ${ }^{1}$, Jun Dai ${ }^{1}$, Yekun Deng ${ }^{1}$, Hong Zhang ${ }^{3}$ and Xiaozhong Zhou ${ }^{1}$

\begin{abstract}
Monocyte-derived cells were shown to promote cartilage repair in osteoarthritis. The role of the long non-coding RNA (IncRNA) MM2P in this function of monocyte-derived cells remained unexplored. Treatment of RAW264.7 murine macrophages and mouse bone marrow-derived macrophages with IL-4 or IL-13 upregulated MM2P expression, upstream of STAT3 and STAT6 phosphorylation. Specifically, MM2P blocked SHP2-mediated dephosphorylation of STAT3 at Try705 and interacted with the RNA-binding protein FUS. In turn, p-STAT3 increased the Sox9 gene expression. These cells released Sox9 mRNA and protein-containing exosomes, as demonstrated by a transmission electron microscope, nanoparticle tracking analysis, and detection of typical surface markers. Their culture supernatant promoted the differentiation of mouse primary chondrocytes, i.e., upregulated the expression of Col1a2 and Acan genes and promoted the secretion of extracellular matrix components proteoglycan and type II collagen. These effects were mediated by Sox9 mRNA and protein delivered to chondrocytes by exosomes. Together, ex vivo treatment of monocyte-derived cells with IL-4 or IL-13 promoted chondrocyte differentiation and functions through exosomemediated delivery of Sox9 mRNA and protein.
\end{abstract}

\section{Introduction}

Osteoarthritis (OA) is the most common degenerative disorder of the joint, which is mainly attributed to an imbalance between repair and breakdown of the joint tissues or abnormal endochondral ossification due to mechanical loading ${ }^{1,2}$. Cartilage repair is suggested to be crucial to OA, however, the detailed mechanism of cartilage repair remains to be further clarified $^{3-5}$.

Correspondence: Hong Zhang (szzhanghong126@126.com) or Xiaozhong Zhou (zhouxz@suda.edu.cn)

${ }^{1}$ Department of Orthopedics, The Second Affiliated Hospital of Soochow University, Suzhou, Jiangsu 215004, China

${ }^{2}$ Department of Hematology, Suzhou Dushuhu Public Hospital (Dushuhu Public Hospital Affiliated to Soochow University, The First Affiliated Hospital of Soochow University, Dushuhu Branch, Suzhou, Jiangsu 215000, China

Full list of author information is available at the end of the article

Co-first authors: Jinyu Bai, Yingzi Zhang, Xin Zheng

Edited by G. Calin
Chondrocytes are known to be the resident cells responsible for maintaining a dynamic equilibrium between catabolism and anabolism in the extracellular matrix $(\mathrm{ECM})^{6}$. Macrophages accumulate and polarize (M1 or M2) in the synovium and articular cavity during OA development ${ }^{7}$. The activated innate immune cells, especially macrophages, participate in OA progression ${ }^{8}$. Macrophages can be activated by certain biomaterials to release specific cytokines and possess immunomodulatory impacts on in vitro tissue recovery and osteogenic differentiation $^{9-11}$. These findings indicate that macrophages M2 phenotype polarization may promote the articular homeostasis and regeneration in OA.

Long non-coding RNAs (lncRNAs) is a group of nonprotein-coding transcripts containing over 200 nucleotides. Their roles in OA progression and cartilage repair have been emerging ${ }^{12,13}$. A recent study shows an abundant expression of lncRNA MM2P contributes to M2

\section{(-) The Author(s) 2020}

(c) (i) Open Access This article is licensed under a Creative Commons Attribution 4.0 International License, which permits use, sharing, adaptation, distribution and reproduction cc) in any medium or format, as long as you give appropriate credit to the original author(s) and the source, provide a link to the Creative Commons license, and indicate if changes were made. The images or other third party material in this article are included in the article's Creative Commons license, unless indicated otherwise in a credit line to the material. If material is not included in the article's Creative Commons license and your intended use is not permitted by statutory regulation or exceeds the permitted use, you will need to obtain permission directly from the copyright holder. To view a copy of this license, visit http://creativecommons.org/licenses/by/4.0/. 
polarization in macrophages ${ }^{14}$. This discovery reminds whether MM2P could promote cartilage repair through triggering M2 polarization.

Signal transducer and activator of transcription family (STATs) had important roles in M2 polarization ${ }^{15,16}$. STAT3 is a transcription factor belonging to the STATs family that is particularly studied in M2 polarization of macrophages ${ }^{17,18}$. In specific, STAT3 is activated through phosphorylation and undergoes dimerization and nuclear translocation to induce transactivation of target genes ${ }^{19}$. As repressors of STAT3 activation, protein tyrosine phosphatase (PTPs) are acknowledged ${ }^{20}$. Members of PTPs, such as SHP1 and SHP2, modulated the tyrosine dephosphorylation of STAT3 to repress STAT3 activity ${ }^{21}$. STAT3 has also been reported to activate SRY-box transcription factor 9 (SOX9) through binding to its proximal promoter to facilitate SOX9 transcription in chondrocytes $^{22}$. SOX9 is a well-established regulator of cartilage repair and recognized to be a master regulator of several chondrogenic factors, including Col2 $\mathrm{a}^{23}, \mathrm{Acan}^{24}$, and Col1 $\mathrm{a}^{25}$. However, the relationship among MM2P, STAT3, and SOX9 is unclear.

Exosomes, a set of small membrane vesicles with $30-100 \mathrm{~nm}$ in size, can be released by most cells ${ }^{26}$. Exosomes are referred to as key intercellular communicators, since they transfer diverse types of molecules to recipient cells. These molecules include lncRNAs, microRNAs (miRNAs), messenger RNAs (mRNAs), and proteins ${ }^{27}$. Macrophages secret and transmit certain functional proteins and RNAs to affect various kinds of cells, such as fibroblast and epithelial cells ${ }^{28,29}$. Previous research demonstrates that osteoarthritic chondrocytes secret exosome-like vesicles to facilitate the production of IL-1 $\beta$ in macrophages and aggravate OA progression ${ }^{30}$. However, whether macrophage-derived exosomes regulate cartilage repair is unknown. Moreover, whether MM2P regulates the effect of macrophage-derived exosomes on cartilage repair has never been investigated. In this study, we clarify whether MM2P promotes cartilage repair through M2 polarization.

\section{Materials and methods}

\section{Cell culture and reagent}

RAW264.7 cells (ATCC, Rockville, Maryland) were maintained in high-glucose DMEM (HDMEM, Hyclone, Logan, UT) adding $10 \%$ fetal bovine serum (FBS; HyClone) and antibiotics (penicillin-streptomycin; HyClone). The extracted newborn mouse articular chondrocytes were cultivated in HDMEM and used for study after one to three passages. All cell cultures were performed at $37^{\circ} \mathrm{C}$ in a $5 \% \mathrm{CO}_{2}$ atmosphere. For treating cells, the recombinant murine IL-4 and IL-13 were acquired from PeproTech (Rocky Hill, NJ). $20 \mu \mathrm{g} / \mathrm{mL}$ of ActynomicinD (ActD) from Sigma-Aldrich (St. Louis,
MO) was applied to detect mRNA stability. $10 \mathrm{mg} / \mathrm{mL}$ of RNase A and 0.5\% Triton-X 100 were both acquired from ThermoFisher Scientific (Waltham, MA).

\section{Isolation and differentiation of bone marrow-derived macrophages (BMDMs)}

BMDMs were obtained obeying former descriptions ${ }^{31}$. C57BL/6 mice (6-week-old) were killed via cervical dislocation and then sterilized using $75 \%$ ethanol. After incising the skin at the root of the hind legs, muscle tissues were removed using scissors from the bone. Later, the bones cut from both ends were kept on ice and were rinsed with a macrophage complete medium (Gibico, Grand Island, NY, USA) using a 30-gauge needle in a sterile environment. The harvested cells were washed and then pipetted up and down in a culture dish to obtain a uniform suspension.

The bone marrow cells were allowed to differentiate into macrophages for 7 days in a culture medium at $37^{\circ} \mathrm{C}$ with $5 \% \mathrm{CO}_{2}$. The medium was changed every $48 \mathrm{~h}$. The protocols for experiments on animals were approved by the ethics committee of the Second Affiliated Hospital of Soochow University. The percentage of markers of BMDMs was measured for further experiments. BMDMs were incubated in a blocking solution containing PBS, $0.1 \%$ Triton-X, $0.1 \%$ Tween-20, $2 \%$ bovine serum albu$\min$, and $4 \%$ goat serum to prevent the non-specific binding for $1 \mathrm{~h}$ at room temperature. After removed the blocking solution, BMDMs were incubated with primary antibodies (anti-F4/80, anti-CD86, and anti-CD206, Abcam) at 1:200 dilutions for $16 \mathrm{~h}$ at $4{ }^{\circ} \mathrm{C}$. BMDMs were incubated with the secondary antibodies (Abcam) for $1 \mathrm{~h}$ at room temperature followed by rinsing with PBS. Finally, BMDMs were subjected to the analysis with the BD FACS Calibur cytometer (Becton-Dickinson, Sparks, MD). The percentage of F4/80, CD86, or CD206-positive cells was calculated.

\section{Real-time quantitative PCR (RT-qPCR)}

Total RNA samples were severally isolated by Trizol kit (GIBCO BRL, Gaithersburg, MD) and quantitated via spectrophotometer (Beckman, Fullerton, CA). The BioRad IQ5 thermocycler (BioRad Laboratories, Inc., Hercules, CA) was used to assess SYBR Green fluorescent dye (Invitrogen, Carlsbad, CA, USA) in double-strand DNA for qPCR. Threshold cycles (Ct) of detected RNA were standardized to housekeeping gene GAPDH, ACTB, or $18 \mathrm{~S}$ rRNA for quantification.

\section{Flow cytometry}

Cells were reaped with the scraper, blocked in 3\% BSA for $45 \mathrm{~min}$, and then cultured with PE-conjugated antimouse CD86 (1:200) or PE-conjugated anti-mouse CD206 (at dilution of 1:200) gated with FITC-conjugated anti- 
mouse F4/80 (1:200) in line with the guidelines. About $1 \times$ $10^{4}$ cells in each sample were subjected to analysis of BD FACS Calibur cytometer (Becton-Dickinson, Sparks, MD).

\section{Transfection}

Cells at about $70-80 \%$ confluence were plated in sixwell culture plates and transfected with indicated plasmids for $48 \mathrm{~h}$ by applying Lipofectamine2000 reagent (Invitrogen). The specific short hairpin RNAs (shRNAs) for MM2P $(\mathrm{sh} \# 1 / 2)$ or FUS (sh-FUS), relative negative control (NC; sh-NC), the pcDNA3.1-STAT3, pcDNA3.1SOX9, and empty vectors were all procured from RiboBio (Guangzhou, China).

\section{Western blot}

Total proteins were extracted in RIPA lysis buffer (Beyotime, Shanghai, China) with the protease inhibitor cocktail (Roche, Pleasanton, CA, USA). Protein concentration was determined with the BCA Protein Assay Kit (Pierce, Rockford, IL, USA). Samples were loaded and separated by $10 \%$ SDS-PAGE gels, followed by transfer to polyvinylidene difluoride (PVDF) membranes (Millipore, Bedford, MA, USA). The membranes were blocked in 5\% skimmed milk and then incubated with indicated primary antibodies and secondary antibody (Abcam, Cambridge, UK). At length, the signals in membranes were observed with the chemiluminescence (ECL) system (BioRad lab, Hercules, CA, USA).

\section{Immunofluorescence (IF)}

Cells were first plated on culture slides for $14 \mathrm{~h}$. Then cells were fixed with $4 \%$ (vol/vol) paraformaldehyde-PBS, permeabilized with $0.5 \%$ Triton-X 100-PBS, and blocked with 5\% (wt/vol) BSA-PBS for $1 \mathrm{~h}$. The slides were incubated with the specific primary antibodies for pSTAT3 and STAT3, followed by rinsing with PBS and stained with secondary antibodies. Fluorescent imaging was performed with a confocal laser-scanning microscope (Leica Microsystems, Wetzlar, Germany).

\section{Measurement of cytokines}

The supernatant was collected from chondrocytes, glycosaminoglycan (GAG) production, and type II collagen (Col II) secretion were separately monitored by colorimetric assay kit (Millipore) and enzyme-linked immunosorbent assay kit (ELISA; BD Biosciences, San Jose, CA) following their relative manual.

\section{Isolation of exosomes and morphology size analysis}

The RAW264.7 cells at 70\% confluence were reaped and rinsed in PBS, then incubated for $48 \mathrm{~h}$ with $10 \%$ exosomedepleted FBS. The exosomes derived from RAW264.7 cells were named as RAW264.7 Exo. The morphology of exosomes was detected by Transmission electron microscopy (TEM) from Hitachi (Tokyo, Japan) and imaged by a digital camera (Olympus, Tokyo, Japan). The size and number of the exosomes were analyzed employing Nanoparticle Tracking Analysis (NTA) with Tunable Resistive Pulse Sensing (Christchurch, New Zealand).

\section{Establishment of animal model}

As previously described, male C57BL/6 mice (2-months old) obtained from the Institute of Laboratory Animals Science (Beijing, China) were subjected to destabilization of the medial meniscus (DMM) surgery to establish the OA model ${ }^{32}$. The animal study followed the principle of randomization. Briefly, the mice were prepared for the surgery through intraperitoneal anesthesia with chloral hydrate $(400 \mathrm{mg} / \mathrm{kg})$. The right knee joint was exposed to the medial capsular incision. Meanwhile, a sham operation was performed as a control group. To assess the role of exosome-derived lncRNA MM2P, the OA mice model were randomly divided into four groups (PBS, Exo, Exo/ sh-NC, and Exo/sh-MM2P). One week after DMM surgery, mice were treated through tail-vein injection. Three weeks later, the mice were killed by cervical dislocation and knee joints were collected for H\&E staining or Saffron O staining as previously elucidated ${ }^{6,33}$.

\section{Chromatin immunoprecipitation (ChIP) analysis}

ChIP assay was performed in light of the protocol of EZ ChIP $^{\mathrm{TM}}$ Chromatin Immunoprecipitation Kit for cell line (Millipore). The treated cells were fixed in formaldehyde for crosslink and sonicated for shearing DNA, followed by incubation with indicated antibodies for $6 \mathrm{~h}$. Normal mouse IgG acted as the NC. The precipitates from beads were analyzed by RT-qPCR.

\section{Dual-luciferase reporter assays}

The amplified SOX9 promoter by PCR was inserted into the firefly plasmid in the pGL3-Basic vector (Promega, Madison, WI). Cells in 96-well plates were co-transfected with a pGL3-SOX9 promoter, pRL-TK-Renilla (Promega), and indicated transfection plasmids for $48 \mathrm{~h}$. The relative luciferase activity was determined by the ratio of firefly to Renilla activity using the Dual-Luciferase Reporter Assay System (Promega).

\section{Exosome labeling}

Exosomes were labeled using PKH67 Green Fluorescent Cell Linker Kit (Sigma-Aldrich) as per the instruction. The exosome pellets were cultured with $2 \mu \mathrm{L}$ PKH67 for $6 \mathrm{~h}$, then ultracentrifuged and rinsed. At length, labeled exosomes were analyzed by a laser-scanning confocal microscope (Leica Microsystems, Wetzlar, Germany). 


\section{Subcellular fraction assay}

Cells were re-suspended in fraction buffer for $10 \mathrm{~min}$ and homogenized to obtain the cytoplasm fraction (supernatant) after centrifugation. The remaining pellets were treated with nuclear isolation buffer for $20 \mathrm{~min}$ on ice for removing the residual cytoplasmic faction. Following RNA extraction from nuclear and cytoplasmic fractions, the cellular location of MM2P was determined by RT-qPCR, with the GAPDH or U6 as cytoplasmic and nuclear references.

\section{RNA pulldown assay}

RNA pulldown assay was implemented in light of the manual of Pierce Magnetic RNA-Protein Pull-Down Kit (ThermoFisher Scientific). Cell lysates were collected for incubation with the indicated biotin-labeled RNA probes, in the presence of beads. After $1 \mathrm{~h}$, the pulldown complex was subjected to RT-qPCR or western blot analysis.

\section{Fluorescence in situ hybridization (FISH)}

MM2P FISH probes constructed at RiboBio (Guangzhou, China) were purchased for FISH assay. Cells fixed in $4 \%$ formaldehyde and washed with $0.5 \%$ Triton-X 100 in PBS. Then, the air-dried cells were hybridized with MM2P FISH probes. After washing and dehydrating, cell nuclei were stained with DAPI (Beyotime), followed by the application of a fluorescence microscope (Olympus).

\section{RNA immunoprecipitation (RIP) analysis}

In strict accordance with the instruction of EZ-Magna RIP kit (Millipore), cells in the RIP lysis buffer were treated with beads conjugated to specific antibodies and anti-IgG as NC and finally assayed by RT-qPCR.

\section{Co-immunoprecipitation (Co-IP)}

Cell lysates were acquired from the treated cells in IP lysis buffer for incubation with a specific antibody to SHP1 or SHP2 and anti-IgG (negative control) all night at $4{ }^{\circ} \mathrm{C}$. Following washing thrice in IP lysis buffer, samples were boiled in SDS loading buffer and eluted for western blot.

\section{Statistical analyses}

In this study, triplicates were required for all assays. Data analyses were conducted by $t$-test or one-way analysis of variance applying PRISM 6 (GraphPad, San Diego, CA), normalized to the threshold of $p$-value $<0.05$. Continuous variables were shown as mean \pm standard deviation (SD).

\section{Results \\ MM2P facilitated M2 polarization}

To confirm the function of MM2P in M2 polarization of RAW264.7 cells, $10 \mathrm{ng} / \mathrm{mL}$ IL-13 or IL-4 were applied to induce the M2 phenotype and the ratio of $\mathrm{F} 4 / 80^{+} \mathrm{CD} 206^{+}$ RAW264.7 cells increased gradually at 24,48 , and $72 \mathrm{~h}$ (Fig. 1a and Supplementary Fig. S1A). MM2P was upregulated following the induction of M2 polarization (Fig. $1 \mathrm{~b}$ and Supplementary Figs. S1B and S2A). Transfection of sh-MM2P\#1/2 downregulated MM2P (Fig. 1c and Supplementary Figs. S1C and S2B), and the ratio of F4/ $80^{+} \mathrm{CD}_{206^{+}}$was decreased (Fig. 1d and Supplementary Fig. S1D). In addition, M2-related genes, including Fizz-1, Arg1, YM1, MRC1, and PPAR- $\gamma$ were downregulated under the silence of MM2P (Fig. 1e and Supplementary Figs. S1E and S2C). The expression of p-STAT3 (Y705) rather than p-STAT3 (S727) was increased under the treatment of IL-4 and IL-13. Nevertheless, there was no significant change in STAT1 and STAT6 (Fig. If and Supplementary Fig. S1F). As showed in Fig. 1g and Supplementary Fig. S1G, increased intensity of p-STAT3 was counteracted by the depletion of MM2P (Fig. 1g and Supplementary Fig. S1G).

IL-4 induced M2 phenotype, whereas LPS induced the M1 phenotype in BMDMs (Fig. 2a). IL-4 increased, whereas LPS reduced the MM2P level (Figs. 2b and 3a). The knockdown of MM2P reduced the ratio of F4/ $80^{+} \mathrm{CD}_{206^{+}}$, whereas overexpression of MM2P increased (Fig. 2d). M2-related genes were downregulated by MM2P knockdown in IL-4-treated BMDMs and increased by MM2P overexpression in LPS-treated BMDMs (Figs. 2e and $3 \mathrm{c}-\mathrm{g}$ ). Moreover, we found that the level of p-STAT3 (Y705) and p-STAT6 showed similar consequences (Fig. 2f). These results indicated that MM2P facilitated M2 polarization through STAT6 and STAT3 signaling.

\section{Exosomes from M2 phenotype macrophages promoted chondrocyte-specific genes and proteins}

RAW264.7 cells induced by IL-13/IL-4 (M2 macrophages) were transfected with sh-MM2P\#1/2. M2 macrophages culture medium $(\mathrm{CM})$ was collected to culture with chondrocytes. Chondrogenic-specific genes were downregulated and differentiation-related gene Col1a1 was upregulated (Fig. 4a and Supplementary Figs. S4A and S5A). The production of sGAG and Col II were reduced (Fig. 4b, c and Supplementary Fig. S4B, C). Similar results were found in BMDMs (Supplementary Figs. S6A-C and S7A-D). By adding the LPS-treated BMDMs CM, chondrogenic-specific genes were downregulated and the differentiation-related gene Colla1 was upregulated in chondrocytes (Supplementary Figs. S6D-F and S7E, F).

Next, to confirm the efficacy of exosomes, exosome-free $\mathrm{CM}$ from M2 macrophage was added to chondrocytes, the expression of chondrogenic-specific markers were decreased significantly compared with exosomal CM (Supplementary Fig. S8A-C). Exosome extracted from M2 macrophage was added into the medium of 


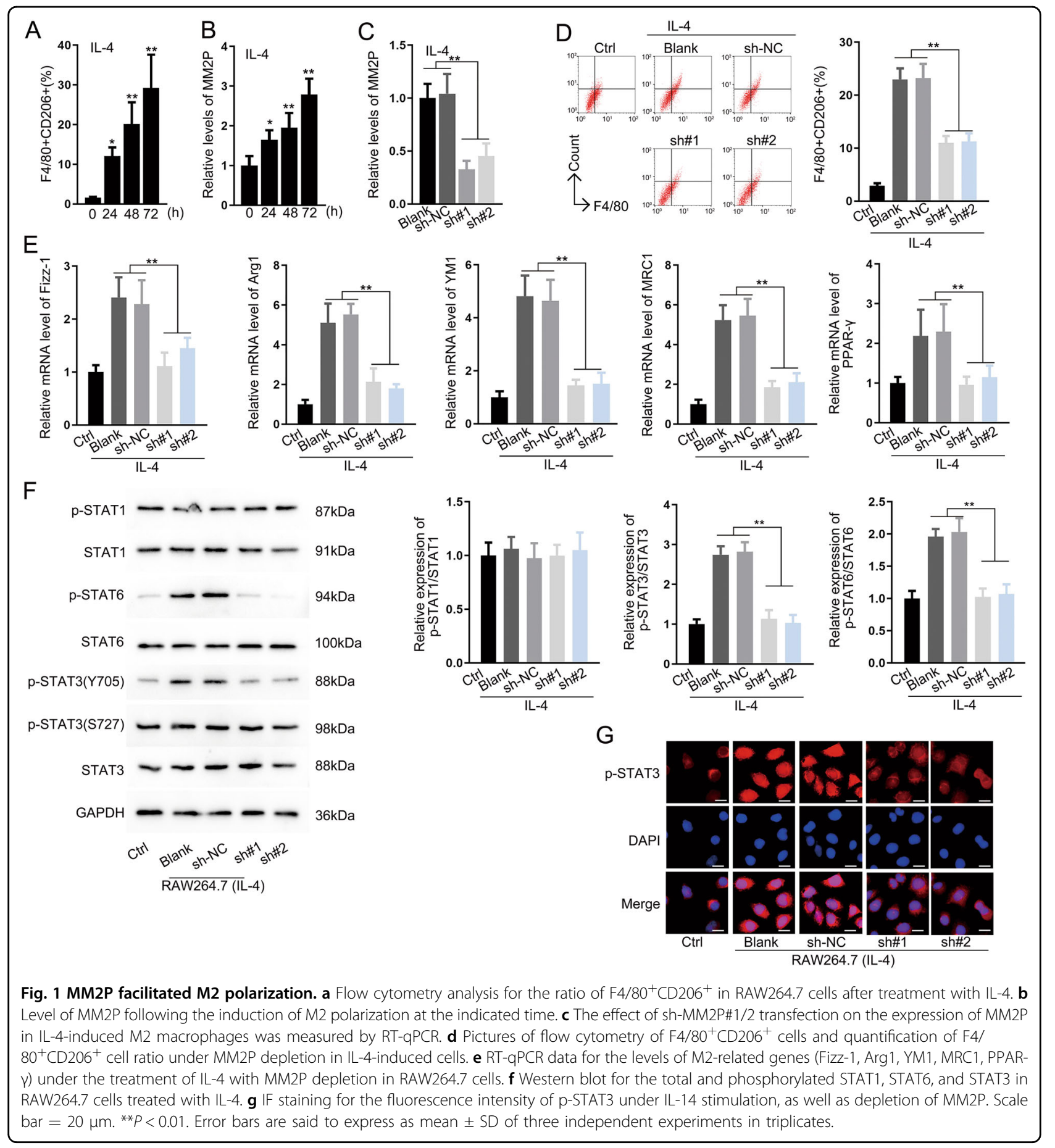

chondrocytes. Chondrogenic-specific markers were increased markedly (Figs. 4f, g and 6e and Supplementary Figs. S5B and S9A-C). We further studied the role of exosome in the animal model. Exosomes injection reversed aberrant subchondral bone formation and sclerosis, whereas MM2P-silenced exosomes revealed no cartilage repair evidence (Supplementary Fig. S3).
MM2P facilitated transactivation of SOX9 in M2 macrophage through STAT3

SOX9 expression was upregulated in M2 macrophages, and MM2P silence inhibited it (Fig. 5a and Supplementary Fig. S10A). Knocking down MM2P reduced the enrichment of STAT3 in the SOX9 promoter biotin group (Fig. 5b). MM2P silence decreased SOX9 abundance in STAT3 


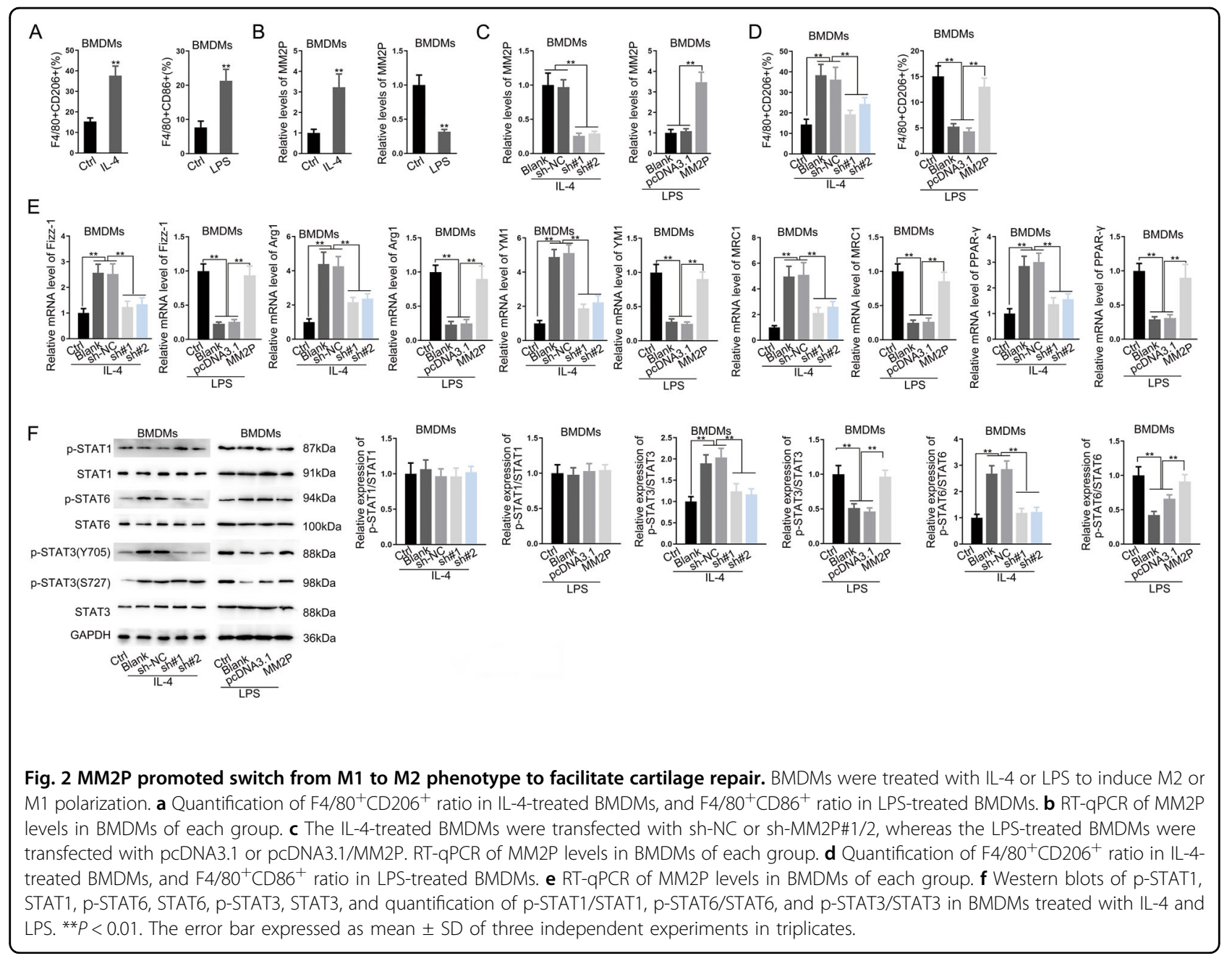

immunoprecipitates (Fig. 5c). Overexpression of STAT3 increased total STAT3 and p-STAT3 (Y705) except for the p-STAT3/STAT3 ratio (Fig. S11A). Luciferase activity of the SOX9 promoter was inhibited by depleting MM2P, reversed by the overexpression of STAT3 (Fig. 5d). STAT3 partially restored the decrease of SOX9 mRNA expression induced by MM2P depletion (Fig. 5e).

We confirmed that the level of SOX9 was higher in exosomes (Fig. 5f). Compared with RNase A treatment, SOX9 expression decreased under the treatment of Triton-X 100 (Fig. 5g). Fluorescence intensity was observed in chondrocytes incubated with exosomes marked by PKH67 (Fig. 5h). We extracted exosomes after transfecting Cy3-labeled SOX9 mRNA and GFP-SOX9 fusion protein into M2 macrophages and co-cultured with chondrocytes. Detecting Cy3-SOX9 and GFP-SOX9 in chondrocytes (Supplementary Fig. S11B). In addition, SOX9 was knocked down in M2 macrophages (Supplementary Figs. S11C and S12A), resulting in the decrease of chondrogenic-specific genes and protein compared with SOX9 normal expression group (Supplementary Figs. S11D-F and S12B, C).

\section{MM2P prevented the SHP2-mediated dephosphorylation of STAT3}

MM2P was mainly located in the cytoplasm (Fig. 6a). Pulldown and RIP analysis verified the binding of STAT3 and MM2P (Fig. 6b, c). MM2P and STAT3 co-localized in the cytoplasm (Fig. 6d). Flag-tagged STAT3 was transfected into M2 macrophages and pulldown analysis did not detect the mutated C terminus STAT3 (Fig. 6e), demonstrating MM2P interacted with STAT3 at the C terminus region. S31-201, the inhibitor of STAT3, reduced the binding between MM2P and STAT3 protein in a dose-dependent manner (Fig. 6f). MM2P knockdown increased the binding between SHP2 instead of SHP1 with STAT3 (Fig. 6g, h). Besides, overexpression of MM2P reduced the enrichment of STAT3 in the immunoprecipitate of SHP2, with total STAT3 and SHP2 unchanged (Supplementary Fig. S13A). Overexpression of MM2P 


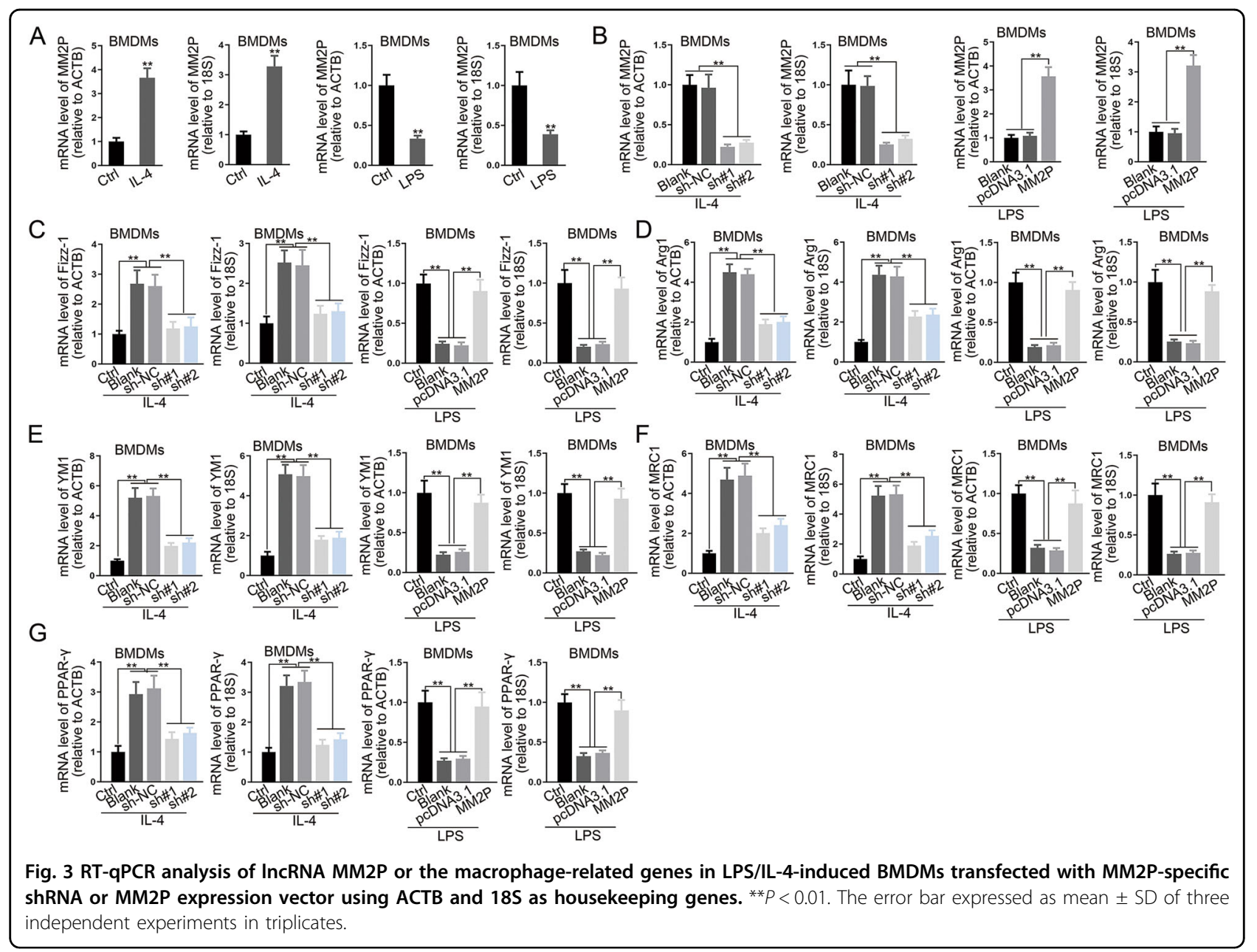

attenuated the colocalization of SHP2 and STAT3 in the cytoplasm, whereas knockdown of MM2P augmented it (Supplementary Fig. S13B). Hence, MM2P prevented the SHP2-regulated dephosphorylation of STAT3.

\section{MM2P interacted with FUS to stabilize SOX9 mRNA}

We identified the protein bands in the pulldown product of MM2P, and found that FUS interacted with MM2P (Fig. 7a and Supplementary Table 1). MM2P enrichment was increased in FUS immunoprecipitates (Fig. 7b). Silencing MM2P reduced SOX9 mRNA enrichment in FUS immunoprecipitates (Fig. 7c). Silencing FUS reduced the abundance of SOX9 mRNA in the MM2P biotin group (Fig. 7d). FUS mRNA and protein levels exhibited no significant change under MM2P depletion (Fig. 7e and Supplementary Fig. S10B). FUS knockdown did not decrease MM2P expression in M2 macrophages (Fig. 7f). CLIP data from Starbase3.0 (http:// Starbase.sysu.edu.cn) identified three potential FUS binding sites on SOX9 mRNA (Fig. 7g). FUS was detectable in the pulldown of SOX9 mRNA with mutation of site 1 and site 2 rather than site 3 (Fig. 7h). FUS interacted with SOX9 mRNA at only site 3 (Fig. 7i). FUS knockdown facilitated SOX9 mRNA degradation with Actinomycin D (Fig. 7j). SOX9 mRNA and protein expression was reduced under the silence of FUS (Fig. 7k and Supplementary Fig. S10C). MM2P knockdown attenuated the mRNA stability of SOX9 (Fig. 7l). In a word, MM2P interacted with FUS to stabilize SOX9 mRNA.

\section{MM2P promote chondrocyte-specific protein through exosome-derived SOX9}

M2 macrophages were transfected with sh-NC, shMM2P\#1, or MM2P\#1 $1^{+}$pcDNA3.1/SOX9. Exosomes were extracted and co-cultured with chondrocytes. SOX9 mRNA and protein expression were increased in the Exo group compared with the PBS group and decreased in Exo/sh\#1 group compared with Exo and Exo/sh-NC groups (Fig. 8a and Supplementary Fig. S10D). Cartilagespecific genes and proteins increased in the Exo group compared with the PBS group. And specific genes and proteins decreased in Exo/sh\#1 group compared with 


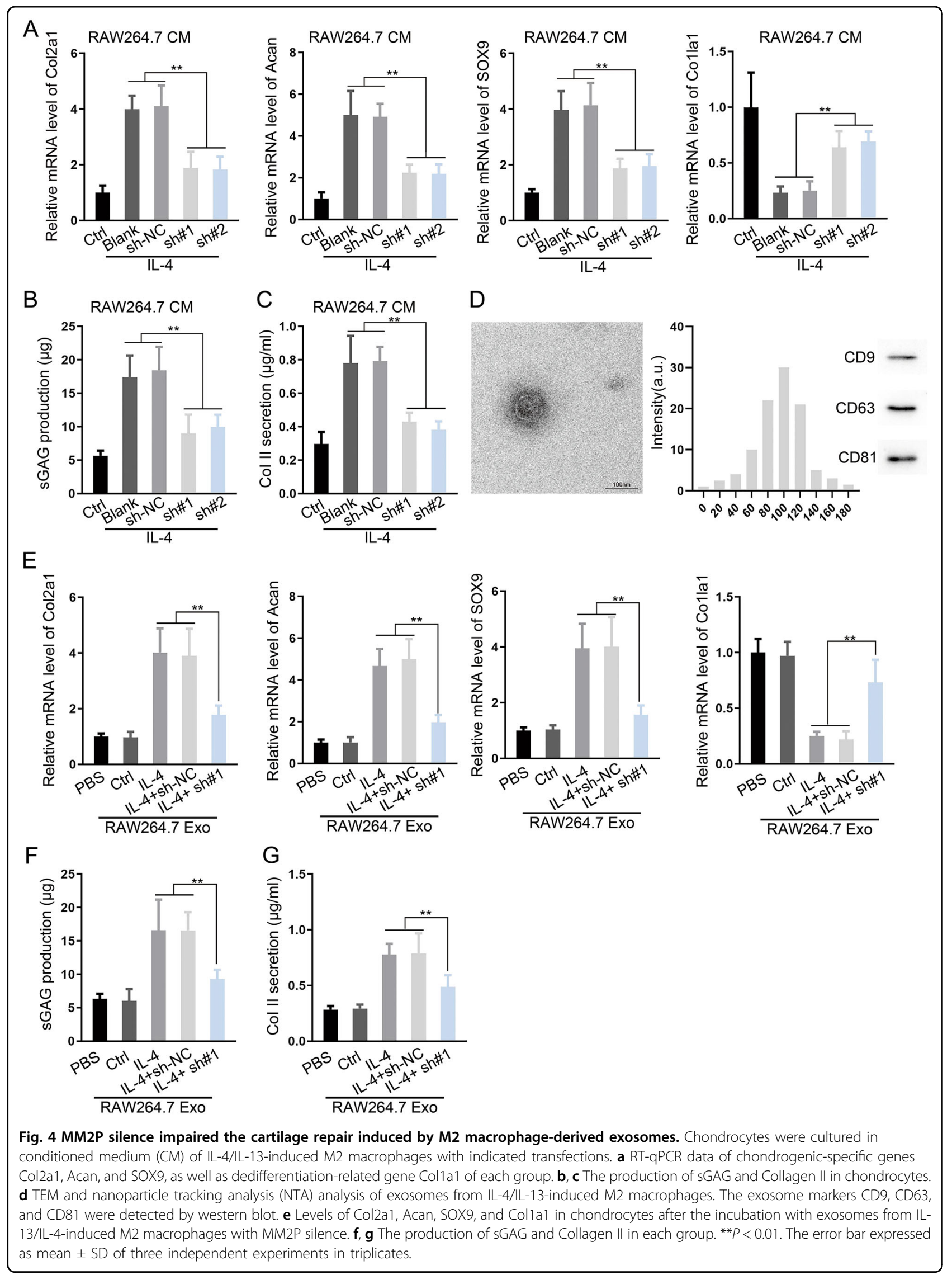




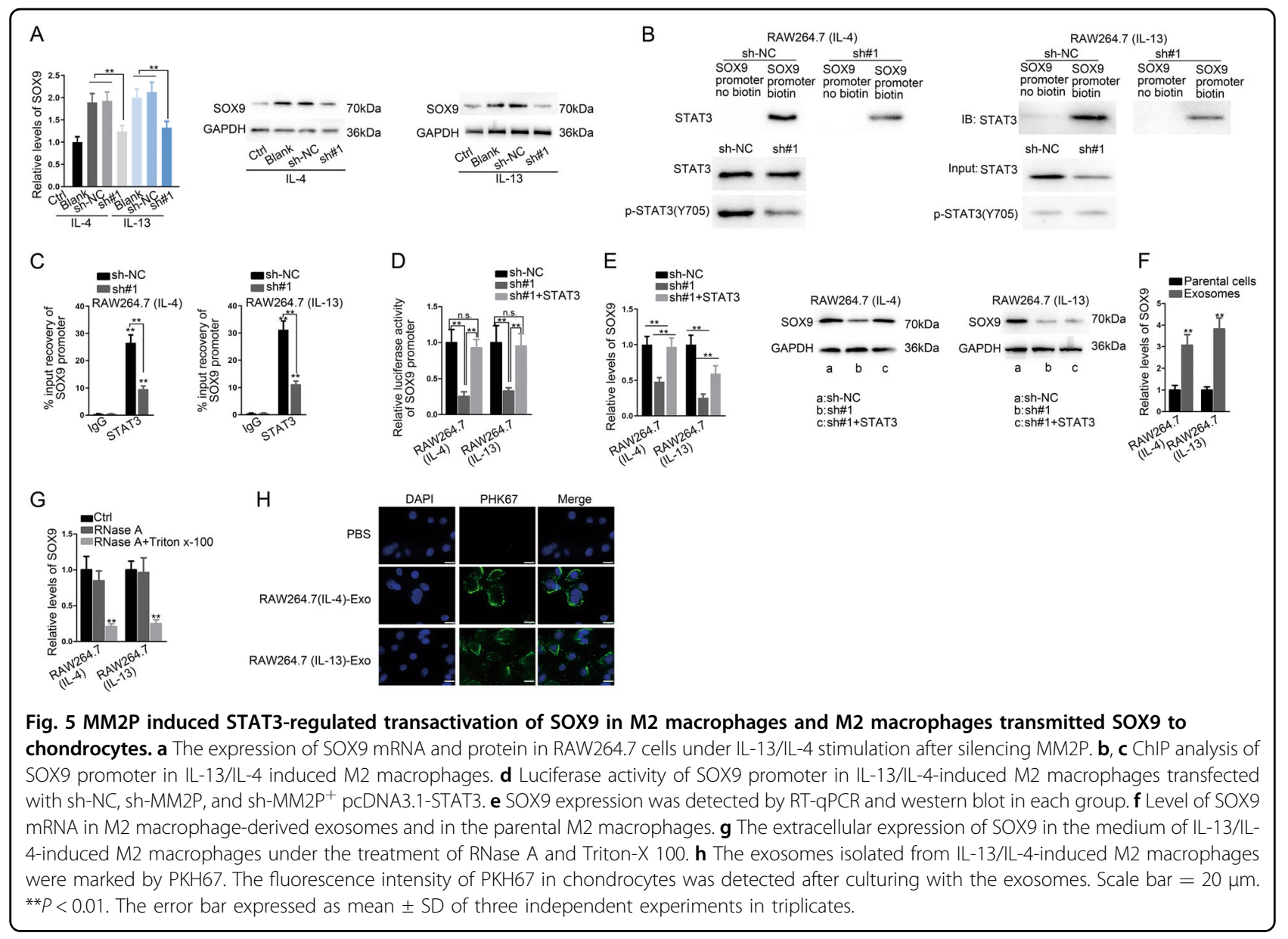

Exo/sh-NC group, which could be partly reversed in Exo/ sh\# $1^{+}$SOX9 group (Fig. 8b-d and Supplementary Fig. $\mathrm{S} 10 \mathrm{E})$. These findings indicated that MM2P increased cartilage-specific genes and proteins to promote cartilage repair.

\section{Discussion}

$\mathrm{OA}$ is a serious disease affecting the health and life quality of the modern population. Although cartilage repair is referred to as a promising approach to treat OA, its mechanism remains elusive ${ }^{3-5}$. In recent years, there is increasing attention on lncRNAs in cartilage repair ${ }^{12,13}$. According to prior research, lncRNA MM2P is identified as the M2 polarization regulator in macrophages ${ }^{14}$. Multiple studies have stated that M2 polarization helped create a pro-chondrogenic atmosphere in the local OA microenvironment ${ }^{9-11}$. Therefore, it is reasonable to deduce that MM2P might positively affect cartilage repair via $\mathrm{M} 2$ polarization.

Our study confirmed that MM2P was upregulated during M2 polarization and contributed to the development of the M2 phenotype, which was concordant to the prior study ${ }^{14}$. It has also been proved that MM2P induced p-STAT6, rather than p-STAT1 during M2 polarization ${ }^{14}$. Besides STAT6 and STAT1, many studies also suggested that STAT3 was important to M2 polarization $^{17,18}$. Interestingly, our study found that MM2P activated not only STAT6 but also STAT3. Specifically, we confirmed that MM2P induced p-STAT3 (Y705) rather than p-STAT3 (S727) (Fig. 1f and Supplementary Fig. S1F), indicating that MM2P also regulated M2 polarization through STAT3 signaling. Since the regulation of MM2P on STAT6 in M2 polarization has been validated, we focused on its impacts on STAT3 signaling in the current study.

Functionally, we proved that M1 macrophages differentiated from BMDMs inhibited the functions of chondrocytes, in which MM2P could promote the M1-to-M2 polarization in BMDMs (Figs. 2e and 3c-g). Recent studies have revealed that exosomes mediated intracellular communication $^{28,29}$. Chondrocytes could release exosome-like vesicles to affect IL-1 $\beta$ production of 


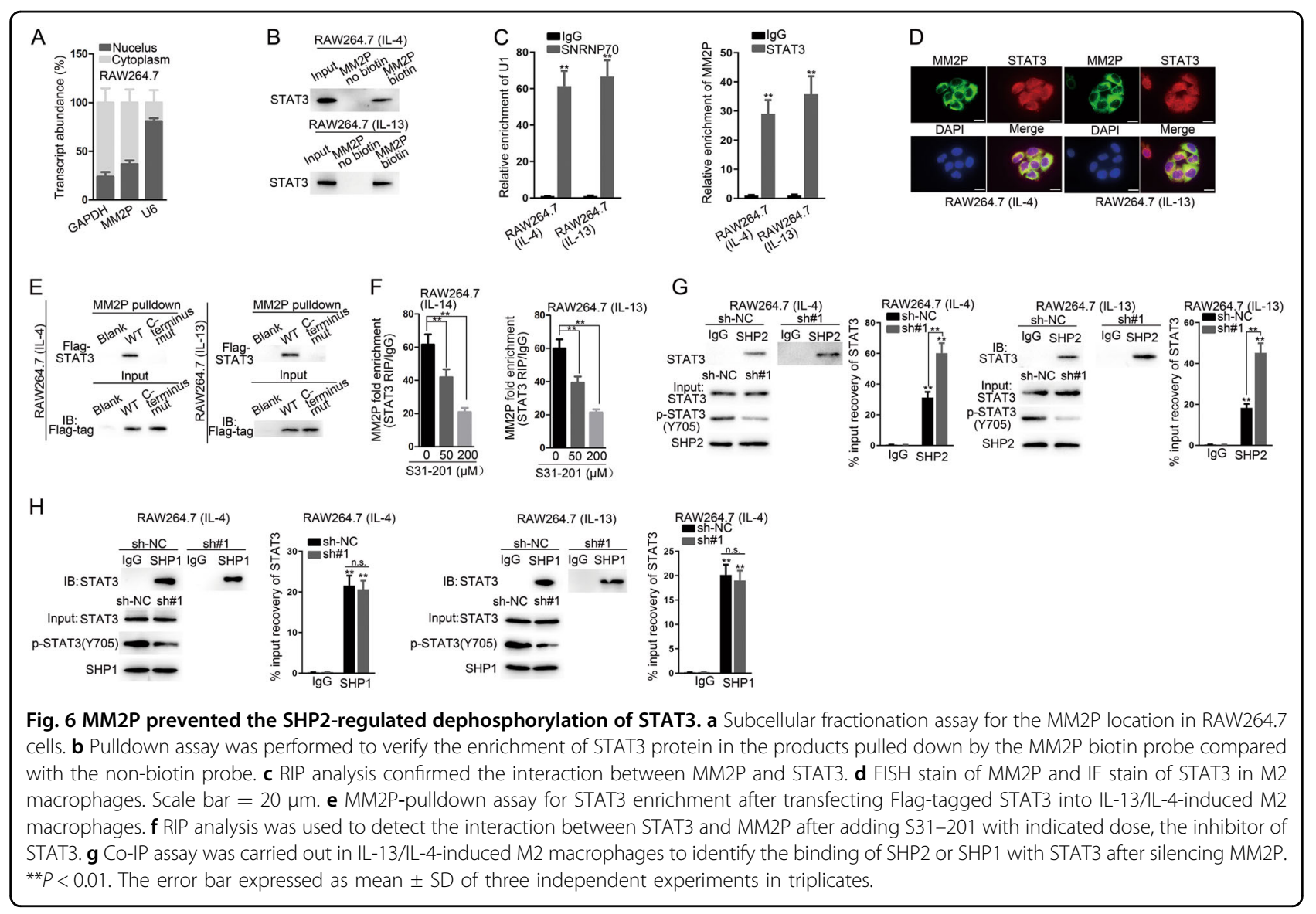

macrophages and aggravate OA progression ${ }^{30}$. Our study showed that M2 macrophages exosome-free CM exhibited much weaker effects on cartilage repair in vitro, indicating that the influence of M2 macrophages on cartilage repair depended on the exosomes partially (Fig. $4 \mathrm{a}-\mathrm{c}$ and Supplementary Fig. S8). We later proved that MM2P mediated the influence of M2 macrophagereleased exosomes on chondrocyte functions, which might potentially promote cartilage repair. In vivo data further supported the results (Supplementary Fig. S3).

A previous study showed that STAT3 could combine to the proximal promoter of SOX9 and trigger its transcription in chondrocytes ${ }^{22}$. SOX9 is axiomatically recognized as the master modulator of many chondrogenic genes, including Acan, Col2a1, and Colla1 ${ }^{23-25}$. Hence, we speculated that MM2P could regulate SOX9 via STAT3, and SOX9 could be transferred by exosomes from M2 macrophages to chondrocytes in cartilage repair. We showed that MM2P demonstrated increased binding and transactivation of STAT3 on SOX9 by inducing pSTAT3 (Y705) (Fig. 5a-e). We also explored the MM2P regulation to p-STAT3 (Y705). A previous study proved that MM2P regulated p-STAT6 by the tyrosine phosphatase inhibitor $\mathrm{Na}_{3} \mathrm{VO}_{4}{ }^{34}$, and suggested that cytoplasmic MM2P might also regulate STAT6 phosphorylation through regulating the SHP1 or SHP2 interaction with STAT6. Another study demonstrated that lnc-DC promoted STAT3 phosphorylation through prohibiting SHP1 via binding to the $\mathrm{C}$ terminus to STAT $^{35}$. SHP1 and SHP2, belong to PTPs, are responsible for the dephosphorylation and inactivation of STAT3 $^{21}$. We verified that MM2P could interact with STAT3 at the $\mathrm{C}$ terminus region that contains Y705 residue $^{36}$ (Fig. 6e). Interestingly, we showed that MM2P inhibited the interaction of STAT3 with SHP2 rather than SHP1 (Fig. 6g, h and Supplementary Fig. S13), suggesting that MM2P regulated SHP2-mediated dephosphorylation of STAT3.

As previously reported, IncRNAs cooperated with RNA-binding proteins to affect target genes ${ }^{37,38}$. Our study first uncovered through pulldown that FUS was a partner with MM2P (Fig. 7a). FUS is a multifunctional protein regulating transcription, RNA splicing, and RNA stability depending on its cellular localization ${ }^{39,40}$. 


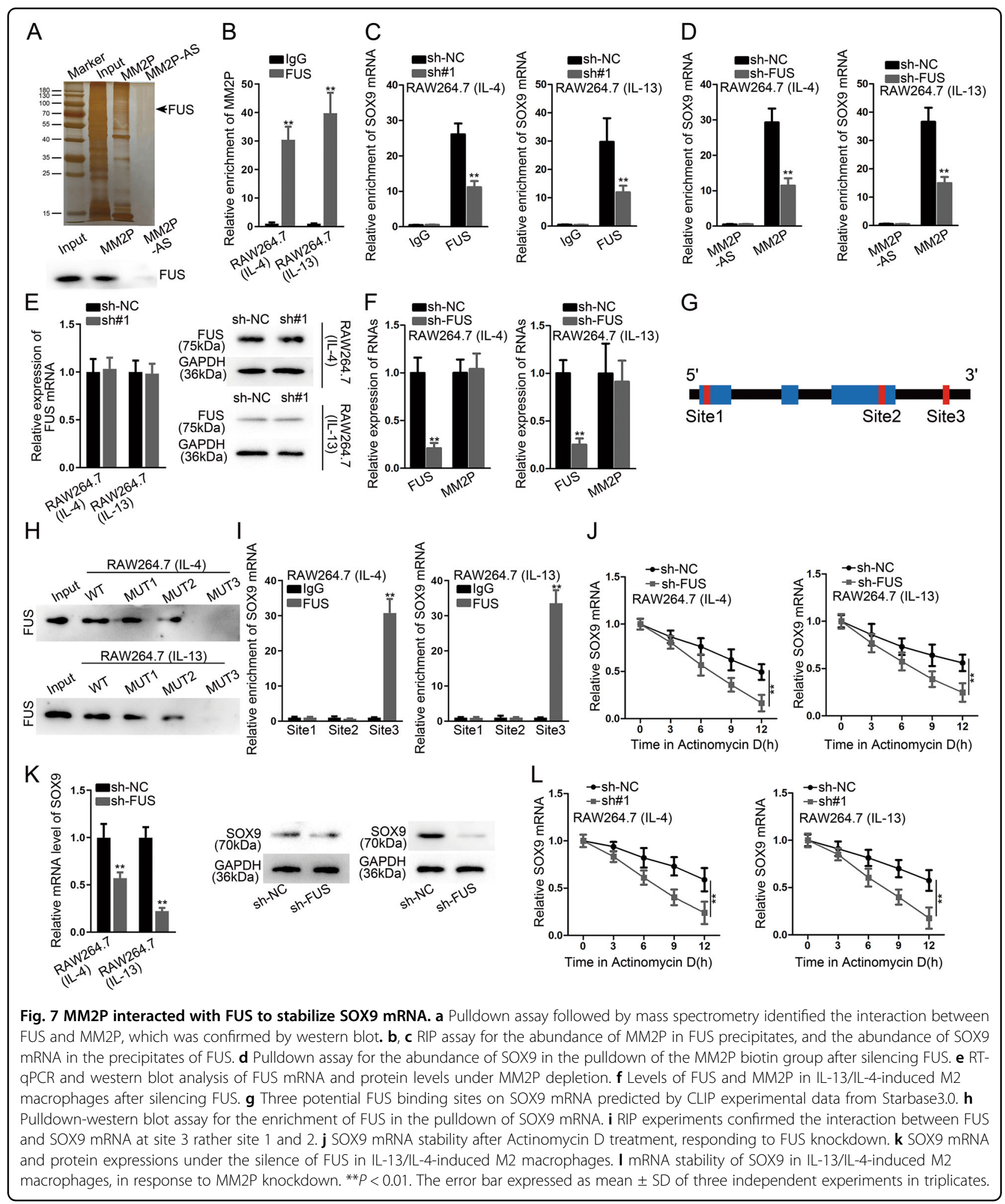

Several studies also delineated that IncRNA coupled with FUS to regulate downstream genes ${ }^{41,42}$. Here, we revealed that MM2P interacted with FUS to stabilize SOX9 mRNA in M2 macrophages. We also showed that
MM2P induced M2 macrophage-derived SOX9 could promote chondrocytes (Fig. 8), indicating that MM2P might influence cartilage repair through SOX9-induced chondrocytes. 


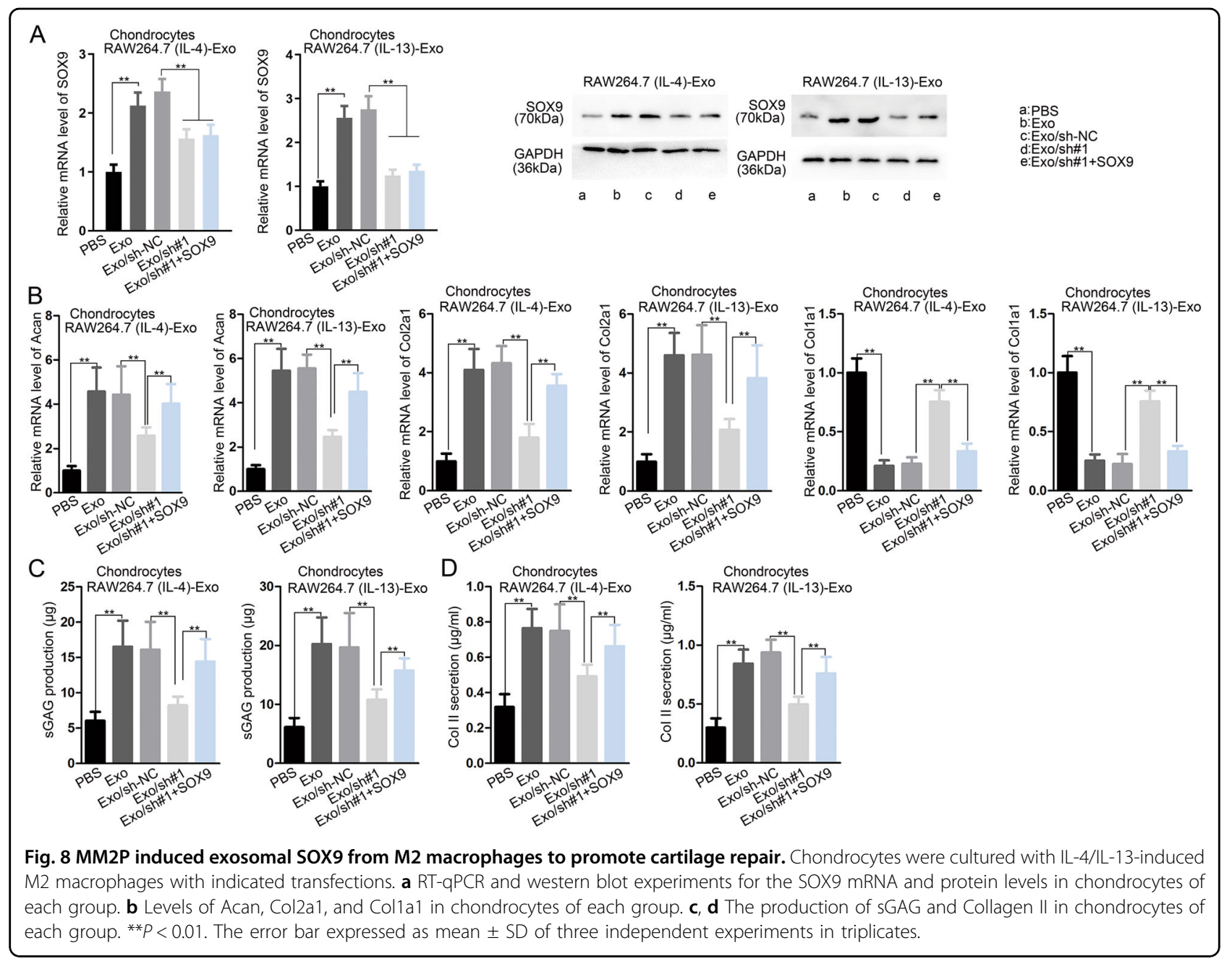

\section{Conclusions}

We demonstrated that IncRNA MM2P significantly enhanced the functions of chondrocytes by inducing M2 macrophage polarization, and promoting the transmission of M2-derived exosomal SOX9 into chondrocytes (Supplementary Fig. S14). Our data clearly illustrated the therapeutic potential of targeting MM2P as a novel promising approach to treat OA through promoting cartilage repair. This study supported a novel therapeutic strategy for OA.

\section{Acknowledgements}

We thank all the members involved in this study. This work was supported by the grants from the National Natural Science Foundation of China (81873995 and 81171712), Clinical Key Diseases Diagnosis and Therapy Special Foundation of Suzhou City (LCZX201904), A-type superior discipline Orthopedics of discipline construction supporting project in the Second Affiliated Hospital of Soochow University (XKTJ-XK202003), the Key Laboratory of Spinal Cord Injury Repair of Suzhou (SZS201807), the National Natural Science Youth Foundation of China (81902207), the Suzhou Young Science and Technology Project (KJXW2018012), Social Development Program of Jiangsu province (BE2019662), Gusu Medical Talents Program of Suzhou (20015), Health Commission of Jiangsu province (H2017066), the Natural Science Foundation of Jiangsu Province (Grant nos. BK20151213 and SBK2019040088), and Jiangsu Province Six Talent Peaks Project (No. SWYY-110).
This study was also supported by the Postgraduate Research \& Practice Innovation Program of Jiangsu Province (KYCX18_2532).

\section{Author details}

${ }^{1}$ Department of Orthopedics, The Second Affiliated Hospital of Soochow University, Suzhou, Jiangsu 215004, China. '2Department of Hematology, Suzhou Dushuhu Public Hospital (Dushuhu Public Hospital Affiliated to Soochow University, The First Affiliated Hospital of Soochow University, Dushuhu Branch, Suzhou, Jiangsu 215000, China. ${ }^{3}$ Department of Obstetrics and Gynecology, The Second Affiliated Hospital of Soochow University, Suzhou, Jiangsu 215004, China

\section{Conflict of interest}

The authors declare that they have no conflict of interest.

\section{Publisher's note}

Springer Nature remains neutral with regard to jurisdictional claims in published maps and institutional affiliations.

Supplementary Information accompanies this paper at (https://doi.org/ 10.1038/s41419-020-02945-5).

Received: 10 October 2019 Revised: 20 August 2020 Accepted: 27 August 2020

Published online: 16 September 2020 


\section{References}

1. Kronenberg, H. M. Developmental regulation of the growth plate. Nature $\mathbf{4 2 3}$, 332-336 (2003)

2. Hiroshi, K. Endochondral ossification signals in cartilage degradation during osteoarthritis progression in experimental mouse models. Mol. Cells 25, 1-6 (2008).

3. Bijlsma, J. W. J., Berenbaum, F. \& Lafeber, F. P. J. G. Osteoarthritis: an update with relevance for clinical practice. Lancet 377, 2115-2126 (2011).

4. Altman, R. D., Schemitsch, E. \& Bedi, A. Assessment of clinical practice guideline methodology for the treatment of knee osteoarthritis with intraarticular hyaluronic acid. Semin. Arthritis Rheum. https://doi.org/10.1016/j. semarthrit.2015.04.013 (2015)

5. Gao, X.-R., Chen, Y.-S. \& Deng, W. The effect of vitamin D supplementation on knee osteoarthritis: a meta-analysis of randomized controlled trials. Int. J. Surg. 46, 14-20 (2017).

6. Zhou, Z. B. et al. circRNA.33186 contributes to the pathogenesis of osteoarthritis by sponging miR-127-5p. Mol. Ther. 27, 531-541 (2019).

7. Zhang, $H$. et al. Synovial macrophage M1 polarisation exacerbates experimental osteoarthritis partially through R-spondin-2. Ann. Rheum. Dis. 77, 1524-1534 (2018)

8. Utomo, L., Bastiaansen-Jenniskens, Y. M., Verhaar, J. A. \& van Osch, G. J. Cartilage inflammation and degeneration is enhanced by pro-inflammatory (M1) macrophages in vitro, but not inhibited directly by anti-inflammatory (M2) macrophages. Osteoarthr. Cartil. https://doi.org/10.1016/j.joca.2016.07.018 (2016).

9. Fearing, B. V. \& Van Dyke, M. E. In vitro response of macrophage polarization to a keratin biomaterial. Acta Biomater. 10, 3136-3144 (2014).

10. Chen, Z. et al. Nanoporous microstructures mediate osteogenesis by modulating the osteo-immune response of macrophages. Nanoscale https://doi. org/10.1039/c6nr06421c (2016).

11. Chen, Z. et al. Osteogenic differentiation of bone marrow MSCs by $\beta$-tricalcium phosphate stimulating macrophages via BMP2 signalling pathway. Biomaterials 35, 1507-1518 (2014).

12. Hu, J. et al. Long non-coding RNA HOTAIR promotes osteoarthritis progression via miR-17-5p/FUT2/ß-catenin axis. Cell Death Dis. 9, 711 (2018).

13. Liu, Q. et al. The TMSB4 pseudogene LncRNA functions as a competing endogenous RNA to promote cartilage degradation in human osteoarthritis. Mol. Ther. https://doi.org/10.1038/mt.2016.151 (2016).

14. Cao, J. et al. LncRNA-MM2P identified as a modulator of macrophage $M 2$ polarization. Cancer Immunol. Res. https:/doi.org/10.1158/2326-6066.cir-180145 (2018).

15. Binnemars-Postma, K., Bansal, R., Storm, G. \& Prakash, J. Targeting the Stat6 pathway in tumor-associated macrophages reduces tumor growth and metastatic niche formation in breast cancer. FASEB J. 32, 969-978 (2017).

16. Lawrence, T. \& Natoli, G. Transcriptional regulation of macrophage polarization: enabling diversity with identity. Nat. Rev. Immunol. 11, 750-761 (2011).

17. Qian, M. et al. Hypoxic glioma-derived exosomes deliver microRNA-1246 to induce M2 macrophage polarization by targeting TERF2IP via the STAT3 and NF-kappaB pathways. Oncogene https:/doi.org/10.1038/s41388-019-0996-y (2019).

18. Giurisato, E. et al. Myeloid ERK5 deficiency suppresses tumor growth by blocking protumor macrophage polarization via STAT3 inhibition. Proc. Natl. Acad. Sci. USA https://doi.org/10.1073/pnas.1707929115 (2018).

19. Nicolas, C. S. et al. The role of JAK-STAT signaling within the CNS. JAKSTAT 2 e22925-e22925 (2013)

20. Murphy, J. M., Tannahill, G. M., Hilton, D. J. \& Greenhalgh, C. J. in Handbook of Cell Signaling 2nd edn. (eds Ralph A. Bradshaw \& Edward A. Dennis) 467-480 (Academic Press, 2010).
21. Lee, J. H. et al. Capillarisin inhibits constitutive and inducible STAT3 activation through induction of SHP-1 and SHP-2 tyrosine phosphatases. Cancer Lett. 345, 140-148 (2014).

22. Hall, M. D. Murray, C. A., Valdez, M. J. \& Perantoni, A. O. Mesoderm-specific Stat3 deletion affects expression of Sox9 yielding Sox9-dependent phenotypes. PLoS Genet. 13, el006610 (2017).

23. Bell, D. M. et al. SOX9 directly regulates the type-ll collagen gene. Nat. Genet. 16, 174-178 (1997)

24. Bar Oz, M. et al. Acetylation reduces SOX9 nuclear entry and ACAN gene transactivation in human chondrocytes. Aging Cell 15, 499-508 (2016).

25. Mori-Akiyama, Y., Akiyama, H., Rowitch, D. H. \& de Crombrugghe, B. Sox9 is required for determination of the chondrogenic cell lineage in the cranial neural crest. Proc. Natl Acad. Sci. USA 100, 9360-9365 (2003).

26. Théry, C., Zitvogel, L. \& Amigorena, S. Exosomes: composition, biogenesis and function. Nat. Rev. Immunol. 2, 569-579 (2002).

27. Tkach, M. \& Théry, C. Communication by extracellular vesicles: where we are and where we need to go. Cell 164, 1226-1232 (2016).

28. Yuan, Z. et al. Macrophages exposed to HIV viral protein disrupt lung epithelial cell integrity and mitochondrial bioenergetics via exosomal microRNA shuttling. Cell Death Dis. 10, 580 (2019).

29. Chen, J. et al. Blockade of IncRNA-ASLNCS5088-enriched exosome generation in M2 macrophages by GW4869 dampens the effect of M2 macrophages on orchestrating fibroblast activation. FASEB J. 33, 12200-12212 (2019).

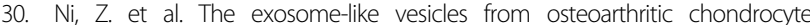
enhanced mature IL-1 $\beta$ production of macrophages and aggravated synovitis in osteoarthritis. Cell Death Dis. 10, 522 (2019).

31. Weischenfeldt, J. \& Porse, B. Bone marrow-derived macrophages (BMM): isolation and applications. CSH Protoc. 2008, pdb.prot5080 (2008).

32. Glasson, S. S., Blanchet, T. J. \& Morris, E. A. The surgical destabilization of the medial meniscus (DMM) model of osteoarthritis in the 129/SvEv mouse. Osteoarthr. Cartil. 15, 1061-1069 (2007).

33. Lin, C., Liu, L., Zeng, C. \& Cui, Z. K. Activation of mTORC1 in subchondral bone preosteoblasts promotes osteoarthritis by stimulating bone sclerosis and secretion of CXCL12. Bone Res. 7, 5 (2019).

34. Cao, J. et al. LncRNA-MM2P identified as a modulator of macrophage M2 polarization. Cancer Immunol. Res. 7, 292-305 (2019).

35. Wang, $P$. et al. The STAT3-binding long noncoding RNA Inc-DC controls human dendritic cell differentiation. Science 344, 310-313 (2014).

36. O'Shea, J. J. \& Plenge, R. JAK and STAT signaling molecules in immunoregulation and immune-mediated disease. Immunity 36, 542-550 (2012).

37. Wang, A. et al. Long noncoding RNA EGFR-AS1 promotes cell growth and metastasis via affecting HuR mediated mRNA stability of EGFR in renal cancer. Cell Death Dis. 10, 154 (2019).

38. Zhao, Y. et al. The IncRNA MACC1-AS1 promotes gastric cancer cell metabolic plasticity via AMPKLLin28 mediated mRNA stability of MACC1. Mol. Cancer 17, 69 (2018).

39. Colombrita, C. et al. TDP-43 and FUS RNA-binding proteins bind distinct sets of cytoplasmic messenger RNAs and differently regulate their posttranscriptional fate in motoneuron-like cells. J. Biol. Chem. 287, 15635-15647 (2012).

40. Ishigaki, S. et al. Position-dependent FUS-RNA interactions regulate alternative splicing events and transcriptions. Sci. Rep. 2, 529-529 (2012)

41. Ge, Z et al. Long noncoding RNA SchLAH suppresses metastasis of hepatocellular carcinoma through interacting with fused in sarcoma. Cancer Sci. 108, 653-662 (2017).

42. $\mathrm{Wu}, \mathrm{H}$. et al. Long noncoding RNA Meg3 regulates cardiomyocyte apoptosis in myocardial infarction. Gene Ther. https://doi.org/10.1038/s41434-018-0045-4 (2018). 